\title{
Discriminación percibida en escolares migrantes en el Norte de Chile
}

\section{Perceived discrimination in migrant schoolchildren in Northern Chile}

\author{
Alejandra Caqueo-Urízar \\ Instituto de Alta Investigación, Universidad de Tarapacá y Centro de Justicia Educacional, Chile. \\ Jerome Flores \\ Escuela de Psicología y Filosofía, Universidad de Tarapacá y Centro de Justicia Educacional, Chile.

\section{Matías Irarrázaval} \\ Departamento de Psiquiatría, Facultad de Medicina, Hospital Clínico Universidad de Chile.
}

\author{
Ninoska Loo \\ Jacqueline Páez \\ Geraldy Sepúlveda \\ Escuela de Psicología y Filosofía, Universidad de Tarapacá y Centro de Justicia Educacional, Chile.
}

Rec (29 de abril de 2019) Acept (6 de mayo de 2019)

\begin{abstract}
Resumen
La migración en Chile ha trascendido enormemente, siendo la educación uno de los principales focos de atención en miras de la transformación. El objetivo de este estudio es describir la discriminación percibida por escolares migrantes de la ciudad de Arica al Norte de Chile, analizando además la relación entre esta última y el estrés por aculturación. Participaron 678 estudiantes de ambos sexos entre Cuarto Básico a Cuarto Medio, entre los 8 y 19 años. Se utilizó la Escala de Discriminación en la Vida Cotidiana (EDS) y la Escala de fuentes de estrés por aculturación (FEAC). Se encontraron diferencias significativas en la discriminación percibida entre escolares migrantes y no migrantes con una puntuación más alta en los migrantes en todas las edades. Se observa también una relación significativa entre discriminación percibida y estrés por aculturación. Se discuten los resultados y sus implicaciones, para el mejoramiento políticas públicas en el ámbito escolar. Palabras Clave: Discriminación percibida; estrés por aculturación; escolares; migrantes; Norte de Chile.
\end{abstract}

\begin{abstract}
Migration in Chile has transcended enormously, with education being one of the main focuses of attention in the face of transformation. The aim of this study is to describe the perceived discrimination by migrant school students from the city of Arica in the North of Chile, also analyzing the relationship between the latter and acculturation stress. 678 schoolchildren of both sexes participated between the Fourth grade to senior year at High School, with ages between 8 and 19. The Everyday Discrimination Scale (EDS) and the Acculturation Stress Source Scale (FEAC) were used. Significant differences were found in perceived discrimination between migrant and non-migrant schoolchildren with a higher score among migrants at all ages.

There is also a significant relationship between perceived discrimination and acculturation stress. The results and their implications are discussed, for the improvement of public policies in the school environment. Keywords: Perceived discrimination; acculturation stress; schoolchildren; migrants; Northern Chile.
\end{abstract}

Correspondencia: Alejandra Caqueo-Urízar, Instituto de Alta Investigación. Universidad de Tarapacá. Antofagasta 1520. Arica, Chile. Phone: 56-58-2205079 - Email: acaqueo@uta.cl

Agradecimientos: Esta investigación fue financiada por CONICYT PIA CIE160007. 


\section{Introducción}

En los últimos 25 años, Chile ha venido exhibiendo una estabilidad económica y política que lo está posicionando entre los principales países receptores de migración en Latinoamérica, observándose un aumento importante de personas que han ingresado al país, de hecho, entre los años 1992 y 2014 se ha visto una cuadruplicación en el número de migrantes residentes en Chile, representando en la actualidad un $2.3 \%$ de la población total. En 12 años se duplicó el porcentaje de población migrante con respecto a la población nacional (Rojas \& Silva, 2016). Este fenómeno impacta en diversas áreas, así, en el ámbito educacional, tanto jóvenes como niños migrantes han ingresado al sistema de educación formal, sin embargo, se observa una falta de precisión en los sistemas escolares que regulen el proceso de integración y se desconoce si se está llevando a cabo de una forma apropiada (Joiko \& Vásquez, 2016).

El número de matrículas de la población infanto-juvenil aumentó 6 veces desde el año 2005 hasta la actualidad, contando con 60.844 migrantes, lo cual corresponde a un $1.7 \%$ de la matrícula total y donde la mayor cantidad de estudiantes migrantes residen en Santiago de Chile y el Norte del país (Joiko \& Vásquez, 2016). Por otro lado, quienes migran a Chile provienen mayoritariamente de países latinoamericanos, concentrándose el mayor porcentaje en países como; Perú (31.7\%); Argentina (16.3\%); Bolivia (8.8\%) y Colombia (3.6\%) (Departamento de extranjería y migración, 2017).

La migración tiene como consecuencias un proceso de aculturación el que implica aprendizaje y adaptación de la persona migrante a la cultura de acogida (Brown, 2017). Esta experiencia puede presentar una serie de efectos negativos que ocurren cuando el niño o niña migrante requiere un poco más de esfuerzo para adaptarse, produciendo así el fenómeno de estrés por aculturación el que se verá incrementado por una posible discriminación percibida por parte de estos niños (Lawton \& Gerdes, 2014; Urzúa, Heredia \& Caqueo-Urízar, 2016).

Este último constructo (discriminación percibida) juega un rol perjudicial en el proceso de migración, el cual hace referencia a aquella percepción personal de ser tratado de forma injusta por tener una identidad étnica diferente, estas experiencias pueden observarse en las relaciones con sus pares, otros adultos o miembros de la misma escuela (Coutinho \& Koinis-Mitchell, 2013).

El rol de la discriminación conlleva, en muchos de los casos, un estigma hacia quien migra por ser parte de una cultura diferente a la que se está introduciendo provocando choques culturales que dificultan el desarrollo de una relación efectiva. Sin duda la discriminación interviene en el trato social que se observa en las escuelas, así, fenómenos como el racismo no han quedado exentos de estos contextos escolares (Riedemann \& Stefoni, 2015; Tijoux, 2013). Enfatizando que las escuelas actúan como un espejo social, que, si bien no pueden controlar el origen étnico de sus estudiantes, sí puede decidir el tipo de mensaje transmitido sobre el valor de la diversidad, esto contribuye directamente en la autopercepción del niño o niña migrante (Suárez-Orozco, 2012). Los efectos de discriminación percibida, en estudiantes pertenecientes a minorías o inmigrantes propiamente tal, no han quedado exentos y se han relacionado con diversa sintomatología: depresión, baja autoestima y estrés post traumático dentro del contexto escolar (Flores, Tschann, Dimas, Pasch \& de Groat, 2010; Sirin, Ryce, Gupta \& Rogers-Sirin, 2013; Tummala-Narra, Alegria \& Chen, 2012; Urzúa, Basabe, Pizarro \& Ferrer, 2017; Yoo \& Lee, 2008).

Si bien, el fenómeno de la migración no es reciente, sólo en los últimos años se ha tomado en consideración cómo afecta este proceso a los migrantes y en específico, a los niños dentro del contexto educativo y cómo los demás afectan a este último en su desarrollo (Urzúa et al., 2017). Aun cuando se observan estudios internacionales que muestran los posibles conflictos o consecuencias que experimentan los migrantes en los países receptores, existe una carencia de estudios en esta línea en población infantil en países Latinoamericanos, como Chile, país donde se ha intentado abordar ésta temática, observándose una serie de limitaciones para crear políticas, programas y sistemas de seguimientos que amparen los derechos de los estudiantes migrantes, que permitan y resguarden el proceso de inserción de estos alumnos a una educación de calidad.

El objetivo de este estudio es describir la discriminación percibida por estudiantes migrantes de 8 a 19 años pertenecientes a escuelas de la ciudad de Arica al Norte de Chile, analizando además la relación entre esta última y el estrés por aculturación. 


\section{Método}

Estudio no experimental cuyo diseño es transeccional correlacional-causal, debido a que se miden todas las variables en un solo momento y se busca establecer relaciones causales entre las variables a partir de la teoría (Hernández, Fernández \& Baptista, 2014).

\section{Participantes}

La muestra inicial contó con 678 escolares de ambos sexos entre Cuarto Año Básico a Cuarto Año de Educación Media, provenientes de diferentes Establecimientos de la ciudad de Arica. El rango de edad fue de 8 a 19 años. Se incluyeron escuelas Municipales, Particular-subvencionadas y Particular-pagadas.

Una vez obtenido el total de migrantes, se seleccionó una muestra equivalente aleatoria entre los no migrantes. El 50\% de los participantes fueron hombres y el otro 50\% fueron mujeres. La edad media fue de 12.4 y la desviación estándar de 2.6 .

\section{Instrumentos}

Escala de Discriminación en la Vida Cotidiana (Everyday Discrimination Scale) (EDS) (Williams, Yu, Jackson \& Anderson, 1997). Instrumento con alta consistencia interna y estructura interna unidimensional que mide la discriminación en la vida cotidiana. En esta escala se pregunta por las veces que los sujetos se han sentido discriminados en su vida. Es una escala tipo Likert de 6 alternativas, desde "casi todos los días" a "nunca". Respecto a sus puntuaciones, la escala original puntúa de manera inversa, es decir, a menor puntuación obtenida, se reconocerá como un mayor índice de discriminación, para efectos de este estudio, las puntuaciones se invirtieron, quedando relacionadas de forma directa, a mayor puntaje mayor índice de Discriminación Percibida. Cuenta con aceptable validez de constructo y adecuada fiabilidad, variando entre 0.8 y 0.9 (Campo, Herazo \& Oviedo, 2015).

Escala de fuentes de estrés por aculturación (FEAC). Cuenta con 17 ítems, de respuesta tipo Likert de 6 opciones. La consigna es: Evalúa si las siguientes situaciones te han sucedido en los últimos seis meses y cuánto te han afectado. Las opciones de respuesta van desde cero ("No he tenido este problema") a 6 ("Me ha afectado mucho"). Las tres dimensiones de la escala son: Añoranza y diferencias con el país de origen: en qué medida los estudiantes extrañan su país de origen ("Extraño a los amigos que tenía en mi país") y lo encuentran diferente del país en que actualmente residen (“Creo que este país es muy diferente al mío”); Adaptación en la Escuela, familia y relación con los pares: los reactivos se centran en la adaptación que los niños, niñas y adolescentes tienen a su escuela ("Me cuesta adaptarme a la escuela"), familia ("Mi familia se pelea más ahora que antes en mi país") y con sus pares ("No me llevo bien con los niños de este país") y Experiencias de discriminación: que aborda diferentes experiencias en que los participantes puedan sentirse discriminados ("A veces me miran mal por ser extranjero"). Actualmente esta escala se encuentra en proceso de validación en Chile, encontrándose un adecuado índice de fiabilidad de 89 (Urzúa, Caqueo-Urízar \& Flores, 2019).

\section{Procedimiento}

1. Aprobación del Comité de Ética de la Universidad de Tarapacá. Este estudio es parte de un proyecto mayor del Centro de Justicia Educacional de la Pontificia Universidad de Chile.

2. Muestreo piloto con 15 estudiantes en la ciudad de Antofagasta para verificar la adecuada comprensión de los ítems. Se solicitó consentimiento a padres y asentimiento a los estudiantes.

3. Se invitó a 42 Establecimientos Educacionales de la ciudad de Arica a participar del estudio. Un 69\% accedió a participar del estudio, considerándose 29 Establecimientos en total.

4. Solicitud de los consentimientos a los padres y posteriormente el asentimiento informado de los estudiantes.

5. Evaluación: Cada aplicación se realizó dentro de 45 minutos en forma grupal en las aulas de los participantes. Al menos dos encuestadores estuvieron presentes para responder las dudas, junto al profesor del mismo establecimiento. Solamente los migrantes responden el instrumento de fuentes de estrés por aculturación. 


\section{Análisis de datos}

Se propone un análisis en cinco etapas. En la primera se presentan los descriptivos demográficos totales de la muestra. En la segunda se realiza un ANOva de dos factores en la muestra total considerando edad y discriminación percibida. En la tercera etapa se analiza una regresión lineal de ser migrante sobre discriminación percibida. En la cuarta, se presenta los motivos a los que atribuyen la experiencia de discriminación, quienes la han experimentado, separando por muestra migrante y no migrante. Finalmente, en la quinta se analiza la muestra migrante considerando sus correlaciones con el total y con cada dimensión del instrumento de fuentes de estrés por aculturación. Se utiliza el programa SPSS versión 21.

\section{Resultados}

\section{Descriptivos generales:}

En el grupo de migrantes la edad media es de 12.4 y la desviación estándar de 2.5. El 52.7\% son hombres y el $47.3 \%$ son mujeres. Se descartaron los instrumentos con más de un $25 \%$ de respuestas en blanco y aquellos con varianza cero.

En el grupo de no migrantes la edad media es de 12.3 y la desviación estándar de 2.7. El 47.3\% son hombres y el $52.7 \%$ son mujeres.

Un 50\% de la muestra está constituida por no migrantes. Entre los migrantes, los provenientes de Bolivia son la mayoría con un $23.7 \%$, seguido de Perú con un 16.4\%. El 9.9\% restante se distribuye entre los demás países (Colombia, Venezuela y Ecuador).

La distribución de estudiantes por curso es mayor en los de primaria, con un 17.4\% en cuarto básico, un $14.9 \%$ en quinto básico y un $15.2 \%$ en sexto básico. En los cursos de secundaria la distribución varía entre un $11.5 \%$ en séptimo básico y un $5.8 \%$ en cuarto medio.

La media de la escala de percepción de discriminación en niños y niñas migrantes fue de 11.8 (DT: 10.4) y la media de niños y niñas no migrantes fue de 8.3 (DT: 9.0). Estas diferencias fueron significativas $[F=10.410$; $p=.000]$.

\section{ANOVA factorial de edad y estatus migrante sobre discriminación percibida:}

Se realizó un ANOva entre grupos (migrante/No migrante) y edad, considerando como variable dependiente la discriminación percibida. Se encuentra un efecto principal significativo de status migrante sobre discriminación percibida $[F(1.678)=6.200, p=.013]$. No se encuentra efecto principal de edad ni de interacción.

En el gráfico 1 se observa que los migrantes tienen mayores medias de discriminación percibida en todas las edades. Si bien su magnitud es mayor, su variación a lo largo del tiempo es similar al de los no migrantes, excepto a la edad de 11 años.

Regresión lineal de ser migrante sobre discriminación percibida:

Se estimó la regresión lineal de estatus migrante sobre discriminación percibida, encontrándose un efecto significativo, $R^{2}=0.32, F(1.678)=22.1, p=.000$. Por tanto, el estatus migrante influencia la discriminación percibida.

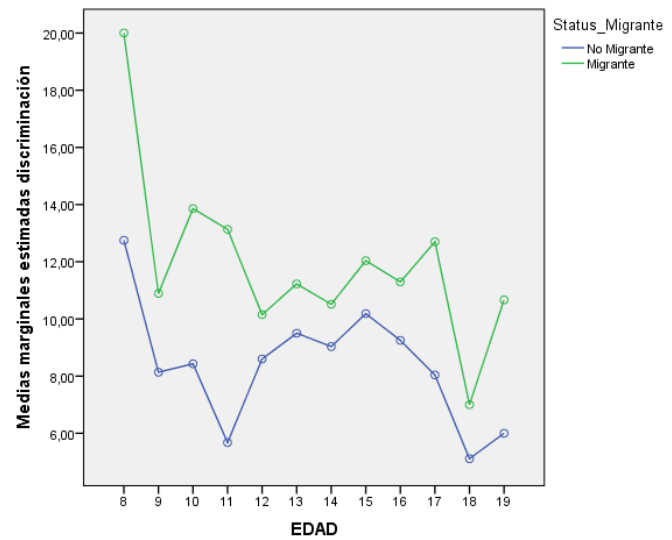

Gráfico 1. Perfil de la interacción entre estatus migrante y edad para la variable discriminación percibida. 
Atribuciones a las experiencias de discriminación:

La tabla 1 muestra una comparación entre los motivos atribuidos a su experiencia de discriminación por los estudiantes de ambas muestras. En la muestra no migrante un $27 \%$ no refirió ninguna experiencia de discriminación. Entre quienes si refirieron estas experiencias destaca que los porcentajes más altos corresponden a estatura o peso y otro aspecto físico. Adicionalmente un $20 \%$ refiere más de un motivo al que atribuye la experiencia.

En la muestra migrante un $18.9 \%$ no refiere experiencias de discriminación. Entre quienes realizaron atribuciones a sus experiencias de discriminación destaca haber experimentado discriminación por sus ancestros o nacionalidad con el porcentaje mayor $(11.8 \%)$ seguido por estatura o peso $(10.3 \%)$ y otro aspecto de la apariencia física $(9.7 \%)$. Un $13.6 \%$ hace atribuciones de más de un motivo.

Tabla 1. Comparación de atribuciones a la experiencia de discriminación en ambas muestras

\begin{tabular}{lcccc}
\hline & \multicolumn{2}{c}{ NO MIGRANTE } & \multicolumn{2}{c}{ MIGRANTE } \\
\hline & Frecuencia & Porcentaje & Frecuencia & Porcentaje \\
\hline Valores perdidos & 2 & 0.6 & 3 & 0.9 \\
Ancestros-nacionalidad & 13 & 3.8 & 40 & 11.8 \\
Género & 10 & 2.9 & 8 & 2.4 \\
Raza & 5 & 1.5 & 31 & 9.1 \\
Edad & 27 & 8 & 31 & 9.1 \\
Religión & 6 & 1.8 & 7 & 2.1 \\
Estatura o peso & 42 & 12.4 & 35 & 10.3 \\
Otro aspecto de la Apariencia física & 49 & 14.5 & 33 & 9.7 \\
Orientación sexual & 0 & 0 & 2 & 0.6 \\
Educación o nivel de ingreso & 22 & 6.5 & 29 & 8.6 \\
Otro & 3 & 0.9 & 10 & 2.9 \\
Más de un motivo & 68 & 20.1 & 46 & 13.6 \\
Sin experiencia. de discriminación & 92 & 27.1 & 64 & 18.9 \\
Total & 339 & 100 & 339 & 100 \\
\hline
\end{tabular}


Relación de estrés por aculturación y discriminación percibida en la muestra migrante:

La tabla 2 muestra la correlación del instrumento total y por dimensiones. Se aprecia que la discriminación percibida correlaciona con el total y con dos dimensiones estrés por aculturación.

Tabla 2. Correlaciones entre discriminación percibida y estrés por aculturación

\begin{tabular}{|c|c|c|c|c|c|}
\hline & $\begin{array}{c}\text { Discriminación } \\
\text { Percibida }\end{array}$ & Añoranza & Adaptación & $\begin{array}{l}\text { Experiencia de } \\
\text { discriminación }\end{array}$ & $\begin{array}{l}\text { Estrés por } \\
\text { Aculturación }\end{array}$ \\
\hline $\begin{array}{l}\text { Discriminación } \\
\text { Percibida }\end{array}$ & 1 & -.006 & $.277^{* *}$ & $.338^{* *}$ & $.259^{* *}$ \\
\hline Añoranza & & 1 & $.464^{* *}$ & $.308^{* *}$ & $.702^{* *}$ \\
\hline Adaptación & & & 1 & $.676^{* *}$ & $.909^{* *}$ \\
\hline $\begin{array}{l}\text { Experiencia de } \\
\text { discriminación }\end{array}$ & & & & 1 & $.812^{* *}$ \\
\hline $\begin{array}{l}\text { Estrés por } \\
\text { Aculturación }\end{array}$ & & & & & 1 \\
\hline
\end{tabular}

Adaptación = Adaptación en la Escuela, familia y relación con los pares, Añoranza= Añoranza y diferencias con país de origen. ${ }^{* *}$ La correlación es significativa al nivel 0,01 (bilateral).

\section{Discusiones}

El objetivo de este estudio fue describir la discriminación percibida por estudiantes migrantes de la ciudad de Arica al Norte de Chile, analizando además la relación entre esta última y el estrés por aculturación.

Los resultados muestran diferencias significativas entre los grupos, es decir, efectivamente los niños, niñas y adolescentes migrantes presentan una percepción de discriminación más alta que los no migrantes. Estas diferencias, además, se presentan en todas las edades. Los resultados de este estudio, en relación a las diferencias entre niños migrantes y no migrantes son similares a los observados en países europeos donde los niños migrantes han debido desarrollar diferentes estrategias de afrontamiento a las empleadas por lo no migrantes (Caqueo-Urízar \& Forns, 2004). Es probable, que al igual que estudios previos, las demandas de adaptación que experimentan los menores migrantes de esta muestra en Chile, se relacionen con conflictos en el contexto próximo donde actualmente se desarrollan (Ayón \& Philbin, 2017). Es posible también que la falta de inclusión existente en el ámbito curricular genere en estos niños y niñas un ambiente con menos espacio y tolerancia a lo culturalmente diferente (Jiménez \& Fardella, 2015), esto último se refleja especialmente y tal como se observa en los resultados, en la vivencia de discriminación por su nacionalidad, lo que está en asociación con la experiencia de estrés por aculturación, donde se observa una estrecha relación entre este constructo y discriminación percibida (Huq, Stein \& González, 2016; Lawton \& Gerdes, 2014).

A pesar de los datos aportados la investigación presenta ciertas limitaciones: El carácter transversal, no permite establecer cuál sería la dirección de las relaciones reportadas, en segundo lugar, la no utilización de otras fuentes de información como la familia o profesores, basándose en el uso exclusivo de autoinforme.

A pesar de estas limitaciones, los resultados son una primera aproximación respecto a la experiencia de discriminación en niños, niñas y adolescentes en el Norte de Chile, considerando la escasez de investigación en esta área.

Futuras investigaciones debieran abordar respecto a posibles intervenciones, tanto a en el ámbito escolar como también en relación a políticas públicas que permitan una mejor inclusión de los estudiantes migrantes, potenciando, tal como lo señala Terriquez (2013), normativas que desarrollen un trato justo, de respeto a cualquier persona y su familia, puesto que ellos influyen directamente en el desarrollo escolar y social del niño o niña migrante. Se debe recordar que la escuela es un microcosmo social, donde la inclusión pueda permitir construir una cultura común. 


\section{Referencias}

Ayón, C., \& Philbin, S. P. (2017). “Tú No Eres de Aquí”: Latino Children’s Experiences of Institutional and Interpersonal Discrimination and Microaggressions. Social Work Research, 41, 19-30. doi.org/10.1093/swr/svw028

Brown, C. S. (2017). School context influences the ethnic identity development of immigrant children in middle childhood. Social Development, 26, 797-812. doi:10.1111/sode.12240

Campo, A., Herazo, E., \& Oviedo, H.C. (2015). Escala de Discriminación en la Vida Cotidiana: Consistencia y estructura interna en estudiantes de medicina. Revista médica Risaralda, 21,39-42. http://dx.doi.org/10.22517/25395203.9129

Caqueo-Urízar, A., \& Forns, M. (2004). Estrategias de afrontamiento en niños inmigrantes escolarizados en Barcelona. En E. Domènech, J. Obiols, M. Jané y S. Subirà (Eds.) Actualizacions en Psicopatologia Clínica (pp. 49-61). Barcelona: Universitat Autònoma de Barcelona.

Coutinho, M. T., \& Koinis-Mitchell, D. (2013). Black Immigrants and School Engagement: Perceptions of Discrimination, Ethnic Identity, and American Identity. Journal of Black Psychology, 40, 520-538. doi:10.1177/0095798413498095

Departamento de Extranjería y Migración (2017). Reportes Migratorios. Población Migrante en Chile. Recuperado desde https://www. extranjeria.gob.cl/media/2017/09/RM_PoblacionMigranteChile1.pdf

Flores, E., Tschann, J. M., Dimas, J. M., Pasch, L.A., \& de Groat, C. L. (2010). Perceived racial/ethnic discrimination, post-traumatic stress symptoms, and health risk behaviors among Mexican American adolescents. Journal of Counseling Psychology, 57, $264-273$. doi:10.1037/a0020026

Hernández, R., Fernández, C., \& Baptista, P. (2014). Metodología de la investigación (6a. ed.). México D.F.: McGraw-Hill.

Huq, N., Stein, G. L., \& Gonzalez, L. M. (2016). Acculturation conflict among Latino youth: Discrimination, ethnic identity, and depressive symptoms. Cultural Diversity and Ethnic Minority Psychology, 22, 377-385. doi: 10.1037/cdp0000070

Jiménez, F., \& Fardella, C. (2015). Diversidad y rol de la Escuela. Discursos del profesorado: en contextos educativos multiculturales en clave migratoria. Revista Mexicana de Investigación Educativa, 20, 419-441. Recuperado desde http://www.scielo.org.mx/scielo. php?script=sci_arttext\&pid=S1405-66662015000200005

Joiko, S., \& Vásquez. A. (2016). Acceso y elección escolar de familias migrantes en Chile: No tuve problemas porque la escuela es abierta, porque acepta muchas nacionalidades. Calidad en la educación, 45, 132-173. doi.org/10.4067/S0718-45652016000200005

Lawton, K. E., \& Gerdes, A.C. (2014). Acculturation and Latino adolescent mental health: Integration of individual, environmental, and family influences. Clinical Child and Family Psychology Review, 17, 385-398. doi:10.1007/s10567-014-0168-0

Riedemann, A., \& Stefoni, C. (2015). Sobre el racismo, su negación, y las consecuencias para una educación anti-racista en la enseñanza secundaria chilena. Polis, 14, 191-216. doi:10.4067/s0718-65682015000300010

Rojas, N., \& Silva, C. (2016). La migración en Chile: breve reporte y caracterización. Observatorio Iberoamericano sobre Movilidad Humana, Migraciones y Desarrollo. Recuperado desde https://www.extranjeria.gob.cl/media/2016/08/informe_julio_agosto_2016.pdf

Sirin, S. R., Ryce, P., Gupta, T., \& Rogers-Sirin, L. (2013). The role of acculturative stress on mental health symptoms for immigrant adolescents: A longitudinal investigation. Developmental Psychology, 49, 736-748. doi.org/10.1037/a0028398.

Suárez-Orozco, C. (2012). Identities under siege: Immigration stress and social mirroring among the children of immigrants. In The new immigration, 149-170: Routledge.

Terriquez, V. (2013). Latino fathers' involvement in their children's schools. Family Relations, 62, 662-675. doi.org/10.1111/fare.12026

Tijoux, M.E. (2013). Las escuelas de la inmigración en la ciudad de Santiago: Elementos para una educación contra el racismo. Polis, 12, 287-307. doi:10.4067/s0718-65682013000200013

Tummala-Narra, P., Alegria, M., \& Chen, C.-N. (2012). Perceived discrimination, acculturative stress, and depression among South Asians: Mixed findings. Asian American Journal of Psychology, 3, 3-16. doi:10.1037/a0024661

Urzúa M,A., Heredia B,O., \& Caqueo-Urízar, A. (2016). Salud mental y estrés por aculturación en inmigrantes sudamericanos en el norte de Chile. Revista Médica De Chile, 144, 563-570. doi:10.4067/s0034-98872016000500002

Urzúa, A., Basabe, N., Pizarro, J., \& Ferrer, R. (2017). Afrontamiento del estrés por aculturación: inmigrantes latinos en Chile. Universitas Psychologica, 16, 221-233. https://dx.doi.org/10.11144/javeriana.upsy16-5.aeai

Urzúa, A., Caqueo-Urízar, A., \& Flores, J. (2019) Propiedades psicométricas de la escala de estrés por aculturación (Manuscrito en preparación).

Williams, D. R., Yu, Y., Jackson, J. S., \& Anderson, N. B. (1997). Racial differences in physical and mental health: Socio-economic status, stress and discrimination. Journal of health psychology, 2, 335-351. https://doi.org/10.1177/135910539700200305

Yoo, H.C., \& Lee, R. (2008). Does Ethnic Identity Buffer or Exacerbate the Effects of Frequent Racial Discrimination on Situational Well-Being of Asian Americans? Journal of Counseling Psychology, 55, 63-74. https://doi.org/10.1037/0022-0167.55.1.63 